\title{
Mode-Scheduling Steering Law of VSCMGs for Multi-Target Pointing and Agile Maneuver of a Spacecraft
}

\author{
Yasuyuki Nanamori \\ School of Science for Open and Environmental Systems, \\ Graduate School of Science and Technology, \\ Keio University, Japan
}

\author{
Masaki Takahashi \\ Department of System Design Engineering, \\ Faculty of Science and Technology, \\ Keio University, Japan
}

\begin{abstract}
This study proposes a method of selecting a set of gimbal angles in the final state and applies the method to the mode-scheduling steering law of variable-speed control moment gyros intended for multi-target pointing manoeuvres in the threeaxis attitude control of a spacecraft. The proposed method selects reference final gimbal angles, considering the condition numbers of the Jacobian matrix of the reaction wheel mode in the final state of a single manoeuvre and that of the constant-speed control moment gyro mode at the start of the upcoming manoeuvre to keep away from the singularities. To improve the reachability of reference final gimbal angles, the nearest set of gimbal angles among nominated sets according to the Euclidean norm is selected as the reference final set at the middle of the single manoeuvre, and then realised by adopting gimbal angle feedback steering logic using null motion. In addition, the manoeuvre profile is designed such that the second half of the single manoeuvre is more gradual and takes longer than the first. Numerical simulation confirms the validity of the proposed method in consecutive manoeuvres.
\end{abstract}

Keywords-Variable-Speed Control Moment Gyros; Attitude Control; Singularity; Steering Law; Spacecraft

\section{INTRODUCTION}

A remote sensing satellite used in an emergency response requires high observation frequency as shown in Figure 1, which is achieved by making highly accurate and large-angle agile manoeuvres consecutively [1,2,3]. To satisfy this requirement, the application of variable-speed control moment gyros (VSCMGs) to an attitude control actuator of a spacecraft and the steering law of the gyros have been studied $[4,5]$. The authors previously proposed a mode-scheduling steering law for VSCMGs, where the suitable set of initial gimbal angles is selected in the constant-speed control moment gyro (CSCMG) mode considering singularity avoidance during an agile attitude manoeuvre, and the steering mode is then transited to the reaction wheel (RW) mode smoothly according to the attitude error of the spacecraft during highly accurate pointing control in the final state of the manoeuvre [6]. Kasai and Kojima proposed the gain-scheduled steering law of VSCMGs [7], focusing on the condition numbers of the Jacobian matrix in an inverse matrix calculation in addition to the consideration of the attitude error. As shown in Figure 2 [7], there is a trade-off relationship between the condition numbers of the Jacobian for the CSCMG mode and that for the RW mode. The method of Kasai and Kojima changes to the gimbal angles for which the condition number of the RW mode is most well-conditioned in the final state of a single manoeuvre, and its validity has been confirmed by numerical simulation.

The present study proposes a method of selecting reference final gimbal angles considering the condition numbers of both the CSCMG mode and RW mode during multi-target pointing manoeuvres, and applies the method to the mode-scheduling steering law of VSCMGs. Through numerical analysis, it is confirmed that the condition number of the wheel Jacobian does not always need to take a minimum value, and a certain value retains the torque generation capability. The proposed method selects the reference final gimbal angles from the predefined nomination to keep not only the condition number of the wheel but also that of the gimbal at a certain level so that the CSCMG mode smoothly ends in the present manoeuvre and drives effectively in the upcoming manoeuvre. Figure 3 shows the sequence of the manoeuvre and steering of VSCMGs adopting the proposed method. Selected reference final gimbal angles are set by gimbal angle feedback steering logic [8] adopting null motion before transition to the RW mode. Since this logic does not guarantee the complete reachability of the reference final angles $[9,10]$, two approaches are proposed to increase the reachability of the gimbal angles. Firstly, when the boundary point is defined as the start of deceleration in a rate profile of a single rest-to-rest manoeuvre, the reference final gimbal angles are selected on the condition that the Euclidean norm between gimbal angles at the boundary point and at each nomination is a minimum.

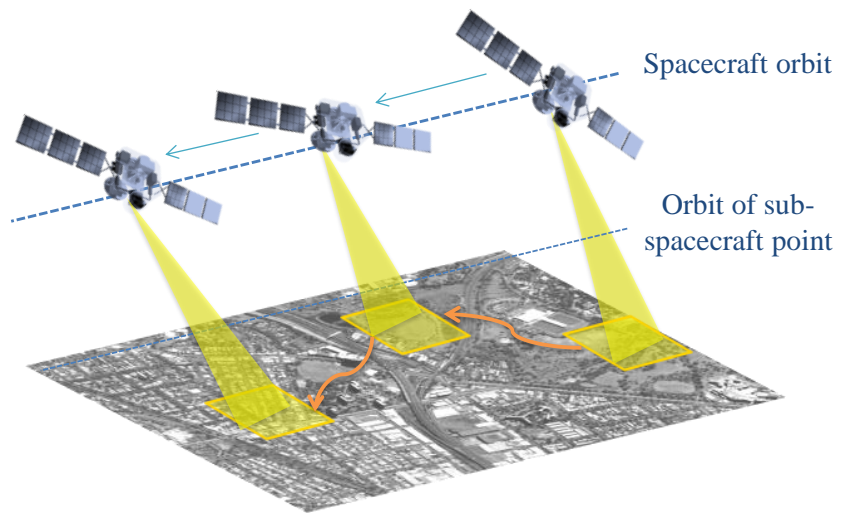

Fig. 1. Image of multi-target pointing manoeuvres 


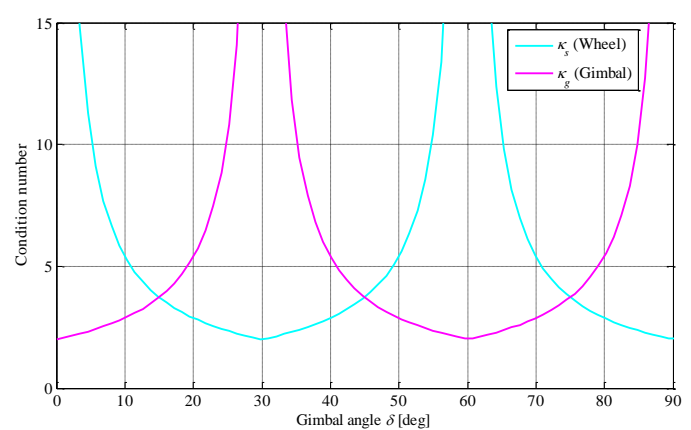

Fig. 2. Condition numbers $\kappa_{s}$ and $\kappa_{g}$ with respect to the gimbal angle [7]

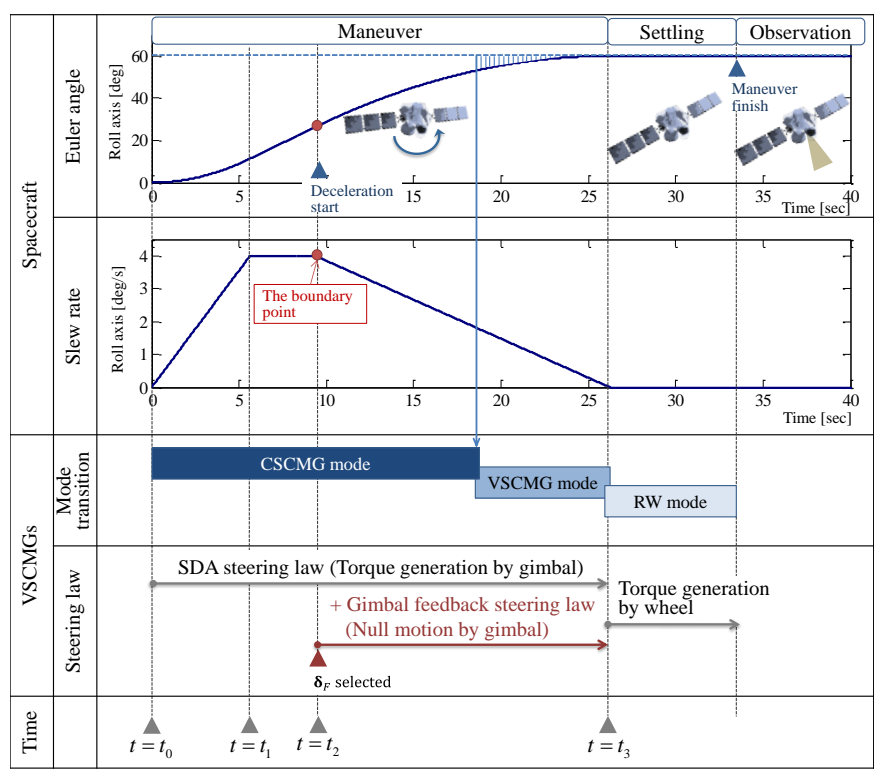

Fig. 3. Sequence of the proposed method

Secondly, a rate profile is designed to make the gimbal angular acceleration more gradual with the intention to having a longer period of gimbal angular feedback after the boundary point. This paper presents the design of the proposed method and carries out numerical simulations to verify the feasibility of the method.

\section{MODELING OF A SPACECRAFT AND VSCMGS}

\section{A. Attitude control of a spacecraft equipped with VSCMGs}

CSCMGs consist of a wheel motor and gimbal motor that constantly rotate a wheel, whereas the VSCMGs control the spin rate of the wheel. Ford and Hall [11] proposed VSCMGs and various singularity avoidance techniques have been developed because the number of degrees of freedom is greater than that of CSCMGs $[12,13]$. The pyramid configuration of $N$ VSCMGs is generally used for the three-axis attitude control of a spacecraft from the viewpoint of hardware redundancy, as shown in Figure 4. The present study deals with the case $N=4$ and a skew angle $\beta=54.7 \mathrm{deg}$. In this paper $\boldsymbol{\delta}$ and $\boldsymbol{\Omega}$ represent the gimbal angles and wheel rotational speed respectively. Firstly, unit direction vectors are defined as
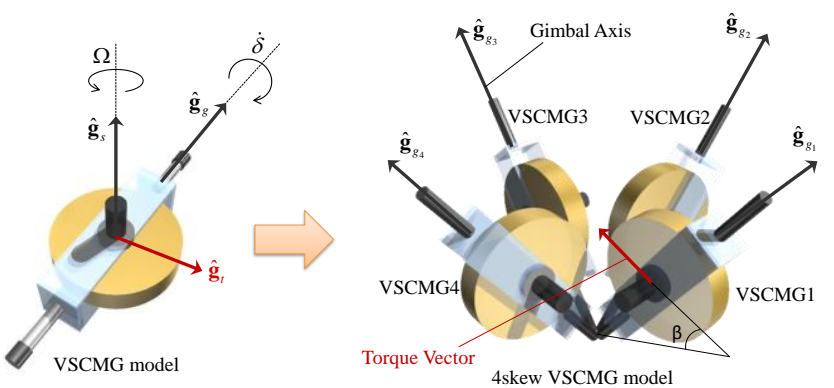

Fig. 4. Skew array of a four-VSCMG system

$$
\begin{aligned}
\boldsymbol{G}_{s} & =\left[\begin{array}{llll}
\hat{\boldsymbol{g}}_{s 1} & \hat{\boldsymbol{g}}_{s 2} & \hat{\boldsymbol{g}}_{s 3} & \hat{\boldsymbol{g}}_{s 4}
\end{array}\right] \\
\boldsymbol{G}_{t} & =\left[\begin{array}{llll}
\hat{\boldsymbol{g}}_{t 1} & \hat{\boldsymbol{g}}_{t 2} & \hat{\boldsymbol{g}}_{t 3} & \hat{\boldsymbol{g}}_{t 4}
\end{array}\right], \\
\boldsymbol{G}_{g} & =\left[\begin{array}{llll}
\hat{\boldsymbol{g}}_{g 1} & \hat{\boldsymbol{g}}_{g 2} & \hat{\boldsymbol{g}}_{g 3} & \hat{\boldsymbol{g}}_{g 4}
\end{array}\right]
\end{aligned}
$$

where, $\hat{\boldsymbol{g}}_{s i}, \hat{\boldsymbol{g}}_{t i}, \hat{\boldsymbol{g}}_{g i}$ is the $i$-th unit direction vector of the spin axis, transverse axis, gimbal axis, respectively.

The inertia matrix of the spacecraft equipped with VSCMGs $\boldsymbol{I}_{B} \in R^{3 \times 3}$ is expressed as

$$
\begin{aligned}
\boldsymbol{I}_{B} & =\boldsymbol{I}_{s}+\sum_{i=1}^{4} \boldsymbol{J}_{i} \\
& =\boldsymbol{I}_{s}+\sum_{i=1}^{4}\left(J_{s i} \hat{\boldsymbol{g}}_{s i} \hat{\boldsymbol{g}}_{s i}{ }^{T}+J_{t i} \hat{\boldsymbol{g}}_{t i} \hat{\boldsymbol{g}}_{t i}{ }^{T}+J_{g i} \hat{\boldsymbol{g}}_{g i} \hat{\boldsymbol{g}}_{g i}{ }^{T}\right)^{\prime}
\end{aligned}
$$

where, $\boldsymbol{I}_{s}$ is the inertia matrix of the spacecraft excluding VSCMGs and $\boldsymbol{J}_{i}$ is the inertia matrix of the $i$-th VSCMG.

Euler's equation of motion of a spacecraft equipped with four VSCMGs is

$$
\boldsymbol{I}_{B} \dot{\boldsymbol{\omega}}=-\tilde{\boldsymbol{\omega}} \boldsymbol{I}_{B} \boldsymbol{\omega}-\boldsymbol{G}_{s} \boldsymbol{\tau}_{s}-\boldsymbol{G}_{t} \boldsymbol{\tau}_{t}-\boldsymbol{G}_{g} \boldsymbol{\tau}_{g}+\boldsymbol{L},
$$

where, $\boldsymbol{\omega} \in R^{3 \times 1}$ is slew rate of the spacecraft and $\boldsymbol{\tau}_{s}, \boldsymbol{\tau}_{t}, \boldsymbol{\tau}_{g} \in R^{3 \times 1}$ are the output torques of the VSCMGs to the wheel spin axis, transverse axis, and gimbal axis respectively. $\boldsymbol{L} \in R^{3 \times 1}$ is the sum of all external torques experienced by the spacecraft. $\tilde{\boldsymbol{\omega}} \in R^{3 \times 3}$ is the skew symmetric form defined as

$$
\tilde{\boldsymbol{\omega}}=\left[\begin{array}{ccc}
0 & -\omega_{3} & \omega_{2} \\
\omega_{3} & 0 & -\omega_{1} \\
-\omega_{2} & \omega_{1} & 0
\end{array}\right] .
$$

\section{B. Steering law of VSCMGs}

1) Mode-scheduling steering law

Neglecting the gimbal inertia effects of the VSCMGs, the relationships among wheel rotational acceleration $\dot{\boldsymbol{\Omega}}$, gimbal rate $\dot{\delta}$ and torque required from the spacecraft attitude control system $\boldsymbol{T}_{r}$ follows that [14] 


$$
\begin{aligned}
\boldsymbol{D}_{0} \dot{\boldsymbol{\Omega}} & +\boldsymbol{D}_{1} \dot{\boldsymbol{\delta}}=\boldsymbol{T}_{r} \\
\boldsymbol{D}_{0} & =\left[\begin{array}{llll}
\hat{\boldsymbol{g}}_{s 1} J_{s 1} & \ldots & \hat{\boldsymbol{g}}_{s 4} J_{s 4}
\end{array}\right] \\
\boldsymbol{D}_{1} & =\left[\begin{array}{llll}
\hat{\boldsymbol{g}}_{t 1} J_{s 1}\left(\Omega_{1}+\omega_{s 1}\right) & \ldots & \hat{\boldsymbol{g}}_{t 4} J_{s 4}\left(\Omega_{4}+\omega_{s 4}\right)
\end{array}\right]
\end{aligned}
$$

where, $\boldsymbol{D}_{0}$ is wheel Jacobian matrix associated with the wheel spin rate and $\dot{\boldsymbol{\Omega}}=\left[\begin{array}{lll}\dot{\Omega}_{1} & \ldots & \dot{\Omega}_{4}\end{array}\right]^{T} \in R^{4 \times 1}, D_{1}$ is gimbal Jacobian matrix associated with gimbal angles and $\dot{\boldsymbol{\delta}}=\left[\begin{array}{lll}\dot{\delta}_{1} & \ldots & \dot{\delta}_{4}\end{array}\right]^{T} \in R^{4 \times 1} . \omega_{s i}(i=1, \cdots, 4)$ is the projection of $\boldsymbol{\omega}$ onto the spin axis of each VSCMG and is expressed as $\omega_{s i}=\hat{\boldsymbol{g}}_{s i}{ }^{T} \boldsymbol{\omega}$. Introducing the state vector $\boldsymbol{\eta} \in R^{8 \times 1}$ and the matrix $Q \in R^{3 \times 8},(5)$ is rewritten as

$$
\begin{aligned}
\boldsymbol{Q} \dot{\boldsymbol{\eta}} & =\boldsymbol{T}_{r} \\
\boldsymbol{\eta} & =\left[\begin{array}{c}
\boldsymbol{\Omega} \\
\boldsymbol{\delta}
\end{array}\right] \\
\boldsymbol{Q} & =\left[\begin{array}{lll}
\boldsymbol{D}_{0} & \vdots & \boldsymbol{D}_{1}
\end{array}\right]
\end{aligned}
$$

It is necessary to solve the weighted pseudo inverse of (6) to calculate the desired $\dot{\boldsymbol{\eta}}$, introducing the weighted diagonal matrix $\boldsymbol{W} \in R^{8 \times 8}$. This inverse kinematics solution is called a steering law and is defined as

$$
\begin{gathered}
\dot{\boldsymbol{\eta}}=\left[\begin{array}{c}
\dot{\boldsymbol{\Omega}} \\
\dot{\boldsymbol{\delta}}
\end{array}\right]=\boldsymbol{W} \boldsymbol{Q}^{T}\left(\boldsymbol{Q W} \boldsymbol{Q}^{T}\right)^{-1} \boldsymbol{T}_{r}, \\
\boldsymbol{W}=\left[\begin{array}{cc}
\boldsymbol{W}_{s} & \boldsymbol{0}_{4 \times 4} \\
\boldsymbol{0}_{4 \times 4} & \boldsymbol{W}_{g}
\end{array}\right], \begin{array}{l}
\boldsymbol{W}_{s}=W_{s} \boldsymbol{I}_{4 \times 4} \\
\boldsymbol{W}_{g}=W_{g} \boldsymbol{I}_{4 \times 4}
\end{array}
\end{gathered}
$$

where, $W_{s}$ denote the weighting function for the wheel angular acceleration, and $W_{g}$ denote that for the gimbal angular velocity. $W_{s}$ and $W_{g}$ are therefore the weights associated with whether the VSCMGs are to perform like RWs or CSCMGs. Introducing these weights allows the control designer to distribute $\dot{\boldsymbol{\delta}}$ and $\dot{\boldsymbol{\Omega}}$, thus realise the required $\boldsymbol{T}_{r}$

In (7) and (8), the transition of the steering mode is then implemented as a sigmoid function of the total sum of the absolute error value $\theta^{\text {error }}$ in the spacecraft three-axis attitude control against the reference attitude, defined by

$$
\begin{gathered}
W_{g}\left(\theta^{\text {error }}\right)=\frac{a}{1+b e^{-c \theta^{\text {error }}}}, W_{s}\left(\theta^{\text {error }}\right)=1-W_{g}\left(\theta^{\text {error }}\right) \\
\theta^{\text {error }}=\sum_{i=1}^{3}\left|\theta_{i}^{\text {ref }}-\theta_{i}\right|
\end{gathered}
$$

where, $\boldsymbol{\theta}^{\text {ref }}$ is the reference attitude angle of the spacecraft after the rest-to-rest manoeuvre and $\boldsymbol{\theta}$ is the Euler angle of the spacecraft at present. $a, b, c$ are arbitrary positive constant values and set as $a=1, b=1808, c=1.5$ in this paper through numerical simulation, with the intention of not being oscillated in the transition of the steering mode and not being saturated in the wheel angular acceleration capacity when settling in the final state of a single manoeuvre. Figure 5 shows the relationship between $\theta^{\text {error }}$ and $W$. According to this function, the actuator drives in CSCMG mode for large-angle manoeuvring and then in VSCMG mode for the intermediate band to prevent the radical hold of the gimbals, and finally, the actuator stops the gimbals and drives in RW mode to settle on the reference attitude angle through the acceleration and deceleration of the wheels.

\section{2) Singularity and condition numbers}

A singularity should be considered when the actuator drives in CSCMG or RW mode. The singular condition occurs when all individual torque vectors are perpendicular to the required torque direction for the specific combination of gimbal angles [15]. This situation means a 'singularity'. In the case of the mode-scheduling steering law defined in (7) distinguishing between the CSCMG mode and RW mode, the singularity of the wheel Jacobian matrix $\boldsymbol{D}_{0}$ and that of the gimbal Jacobian matrix $D_{1}$ should be considered individually [16]. A singularity of the RW mode and CSCMG mode occur when matrices $\boldsymbol{D}_{0}$ and $\boldsymbol{D}_{1}$ meet the conditions respectively

$$
\begin{aligned}
& \operatorname{rank}\left(\boldsymbol{D}_{0}\right)<3 \text { or } \operatorname{rank}\left(\boldsymbol{D}_{0} \boldsymbol{D}_{0}^{T}\right)<3 \Leftrightarrow \operatorname{det}\left(\boldsymbol{D}_{0} \boldsymbol{D}_{0}{ }^{T}\right)=0 \\
& \operatorname{rank}\left(\boldsymbol{D}_{1}\right)<3 \text { or } \operatorname{rank}\left(\boldsymbol{D}_{1} \boldsymbol{D}_{1}^{T}\right)<3 \Leftrightarrow \operatorname{det}\left(\boldsymbol{D}_{1} \boldsymbol{D}_{1}^{T}\right)=0
\end{aligned}
$$

The singular index, also defined as condition number, represents the distance from singularity which is obtained through the singular value decomposition of the Jacobian matrix. The condition number of $\boldsymbol{D}_{0}$ and $\boldsymbol{D}_{1}$ is respectively defined as

$$
\kappa_{s}=\frac{\sigma_{s 1}}{\sigma_{s 3}}, \kappa_{g}=\frac{\sigma_{g 1}}{\sigma_{g 3}},
$$

where, $\sigma_{s i}(i=1 \cdots 3)$ is the singular value of $\boldsymbol{D}_{0}$ ( $\sigma_{s 1} \geq \sigma_{s 2} \geq \sigma_{s 3} \geq 0 \quad$ ), and $\sigma_{g i}$ is that of $\boldsymbol{D}_{1}$ ( $\sigma_{g 1} \geq \sigma_{g 2} \geq \sigma_{g 3} \geq 0$ ) [17].

Larger values of $\kappa_{s}$ and $\kappa_{g}$ indicate that the singularity is closer for each Jacobian matrix. Provided that the spacecraft

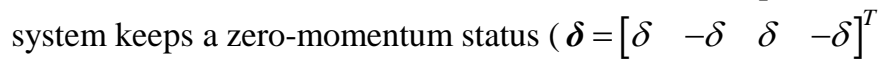
in this paper) when manoeuvring, the relationships between $\delta$ and $\kappa_{s}, \kappa_{g}$ are those shown in Figure 2.

3) Implementing singular-direction avoidance logic to the VSCMG steering law 


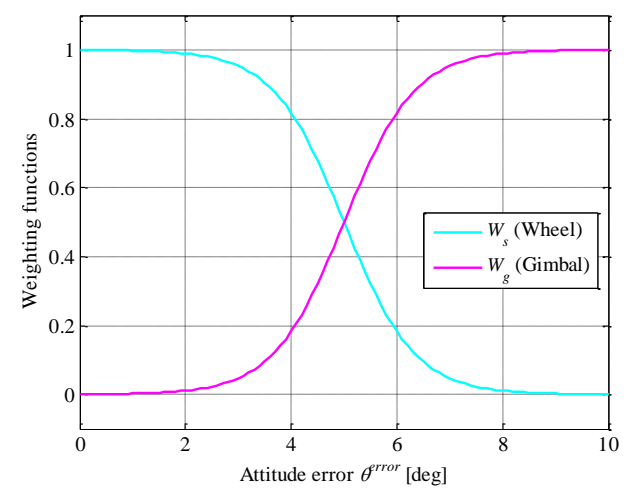

Fig. 5. Weighting functions $\boldsymbol{W}_{s}$ and $\boldsymbol{W}_{g}$ of the steering mode

To avoid a singularity while manoeuvring in the CSCMG mode, the mode-scheduling steering law in (7) applies the singular-direction avoidance (SDA) steering law [17]. By decomposing and adding a singularity avoidance parameter against $\boldsymbol{D}_{1}$, the modified matrix $\boldsymbol{D}_{1 S D A}$ is introduced as

$$
\begin{aligned}
& \boldsymbol{D}_{1 S D A}=\boldsymbol{U}_{g} \boldsymbol{\Sigma}_{g S D A} \boldsymbol{V}_{g}^{T} \\
& \boldsymbol{\Sigma}_{g S D A}=\left[\begin{array}{cccc}
\sigma_{g 1} & 0 & 0 & 0 \\
0 & \sigma_{g 2} & 0 & 0 \\
0 & 0 & \left(\sigma_{g 3}^{2}+\alpha\right) / \sigma_{g 3} & 0
\end{array}\right], \\
& \alpha=\alpha_{0} e^{-\operatorname{det}\left(\boldsymbol{D}_{1} \boldsymbol{D}_{1}^{T}\right)}
\end{aligned}
$$

where, $\boldsymbol{U}_{g} \in R^{3 \times 3}, \boldsymbol{V}_{g} \in R^{4 \times 4}$ are unitary matrices and $\alpha_{0}$ is a design parameter and set as a positive constant. From (7) and (12), the mode-scheduling steering law implementing SDA logic is then expressed as

$$
\begin{aligned}
\dot{\boldsymbol{\eta}}_{S D A} & =\left[\begin{array}{c}
\dot{\boldsymbol{\Omega}} \\
\dot{\boldsymbol{\delta}}_{S D A}
\end{array}\right] \\
& =\left[\begin{array}{c}
\boldsymbol{W}_{s} \boldsymbol{D}_{0}{ }^{T} \\
\boldsymbol{W}_{g} \boldsymbol{D}_{1 S D A}^{T}
\end{array}\right]\left[\boldsymbol{D}_{0} \boldsymbol{W}_{s} \boldsymbol{D}_{0}{ }^{T}+\boldsymbol{D}_{1 S D A} \boldsymbol{W}_{g} \boldsymbol{D}_{1 S D A}{ }^{T}\right]^{-1} \boldsymbol{T}_{r}
\end{aligned}
$$

\section{PROPOSED METHOD}

\section{A. Analysis of the selection of reference final gimbal angles}

Considering the steering of the VSCMGs in consecutive attitude manoeuvres, the reference final gimbal angles of a single attitude manoeuvre are analysed. In the range $0 \leq \delta \leq 30$, which is intentionally limited from the viewpoint of symmetry in Fig. 2, each specification is examined in the three domains $0<\delta<10,10 \leq \delta \leq 20,20<\delta<30$, cantered on $\delta=5,15,25$ respectively. First, in the domain $20<\delta<30$, the behaviour of gimbals could be unstable and it could be difficult to transit to the RW mode smoothly because $\boldsymbol{D}_{1}$ is near singularity. In addition, this state makes gimbals difficult to begin steering in the upcoming manoeuvre. Therefore, $\delta \leq 20$ is preferable from the viewpoint of acquiring the condition number of $\boldsymbol{D}_{1}$. Second, in the domain $0<\delta<10$, the output torque of the RW mode in the final state of manoeuvre could be limited depending on the direction because $\boldsymbol{D}_{0}$ is near singularity. In more detail, $\boldsymbol{D}_{0}$ can be decomposed as

$$
\begin{aligned}
\boldsymbol{D}_{0} & =\boldsymbol{U}_{s} \boldsymbol{\Sigma}_{s} \boldsymbol{V}_{s}^{T} \\
\boldsymbol{U}_{s} & =\left[\begin{array}{lll}
\boldsymbol{u}_{s 1} & \boldsymbol{u}_{s 2} & \boldsymbol{u}_{s 3}
\end{array}\right] \\
\boldsymbol{\Sigma}_{s} & =\left[\begin{array}{cccc}
\sigma_{s 1} & 0 & 0 & 0 \\
0 & \sigma_{s 2} & 0 & 0 \\
0 & 0 & \sigma_{s 3} & 0
\end{array}\right], \\
\boldsymbol{V}_{s} & =\left[\begin{array}{llll}
\boldsymbol{v}_{s 1} & \boldsymbol{v}_{s 2} & \boldsymbol{v}_{s 3} & \boldsymbol{v}_{s 4}
\end{array}\right]
\end{aligned}
$$

where, $\boldsymbol{U}_{s}$ and $\boldsymbol{V}_{s}$ are unitary matrices, $\boldsymbol{u}_{s i}(i=1 \cdots 3)$ are left singular vectors, and $\boldsymbol{v}_{s j}(j=1 \ldots 4)$ are right singular vectors.

From this singular value decomposition, the wheel maximum output torque is expressed as

$$
\boldsymbol{U}_{\text {smax }}=\sigma_{s 1} \boldsymbol{u}_{s 1}+\sigma_{s 2} \boldsymbol{u}_{s 2}+\sigma_{s 3} \boldsymbol{u}_{s 3},
$$

where, each element of $\boldsymbol{U}_{\text {smax }} \in R^{3 \times 1}$ is the maximum output torque around the roll, pitch, or yaw axis respectively. Figure 6 shows the relationship between $\delta$ and $\boldsymbol{U}_{\text {smax }}$. Note that each element of $\boldsymbol{U}_{\text {smax }}$ takes an absolute value. Figure 6 reveals the possibility that the output torque around the yaw axis becomes small as $\delta$ approaches zero. In general, a remote sensing satellite not only often manoeuvres around its roll and pitch axes to orient its mission sensor but also compensates for the attitude errors around the yaw axes to settle using the RW mode. Therefore, $10 \leq \delta$ is preferable from the viewpoint of acquiring the condition number of $\boldsymbol{D}_{0}$. This discussion reveals that, for the reference final gimbal angles, the domain $10 \leq \delta \leq 20$ cantered on $\delta=15$ is preferable considering the trade-off between $\kappa_{s}$ and $\kappa_{g}$ of $\boldsymbol{D}_{0}$ and $\boldsymbol{D}_{1}$. The angles are then set as

$$
\boldsymbol{\delta}_{F}(m)=\left[\begin{array}{llll}
15 m & -15 m & 15 m & -15 m
\end{array}\right]^{T},
$$

where, $m$ is the positive integer and the following numerical simulation adopts $m=1$.

\section{B. Applying gimbal angle feedback steering logic to the VSCMG mode-scheduling steering law}

An overall design of the proposed method along with a time series of a single manoeuvre is shown in Figure 3. In a rate profile of the spacecraft $\omega^{\text {profile }}$, it is supposed that $t_{0}$ is the time at which the manoeuvre begins, $t_{1}$ is the time at which there is a change from acceleration to constant slew, $t_{2}$ is the time at which deceleration begins, and $t_{3}$ is the time at which the required attitude is realised. 


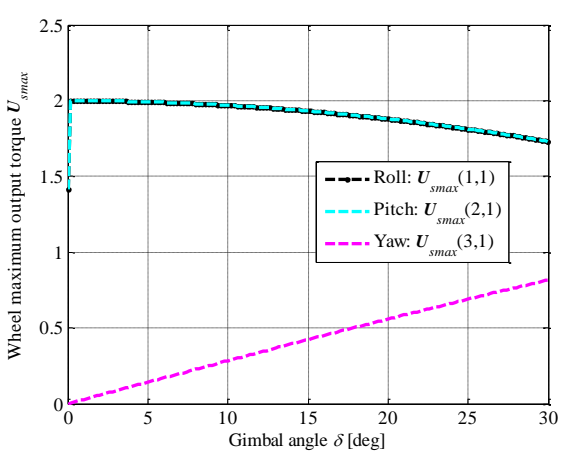

Fig. 6. Wheel maximum output torque $\boldsymbol{U}_{\operatorname{smax}}$

Note that if $\omega^{\text {profile }}$ is triangular rather than having a trapezoidal shape, then $t_{1}=t_{2}$.

At $t \geq t_{0}$ for large-angle manoeuvring, the actuator drives in CSCMG mode and outputs torque by gimbaling. When settling in the final state of a single manoeuvre, the actuator drives in RW mode and compensates for small attitude fluctuations through the acceleration and deceleration of the wheels. To transit to the RW mode with the reference final gimbal angles of a single attitude manoeuvre, gimbal angle feedback steering logic using null motion [8] is applied. This logic allows gimbal angles to be guided to the desired angles without effecting the output torques using null motion. Gimbal angle feedback steering logic is expressed by

$$
\begin{aligned}
& \dot{\boldsymbol{\eta}}_{G F B}=\left[\begin{array}{l}
\boldsymbol{0}_{4 \times 1} \\
\dot{\boldsymbol{\delta}}_{G F B}
\end{array}\right] \\
& \dot{\boldsymbol{\delta}}_{G F B}=K_{N} \boldsymbol{I}_{4 \times 4} \boldsymbol{S}\left(\boldsymbol{\delta}_{F}-\boldsymbol{\delta}\right), \\
& \boldsymbol{S}=\boldsymbol{I}_{4 \times 4}-\boldsymbol{P} \\
& \boldsymbol{P}=\boldsymbol{D}_{1}^{T}\left(\boldsymbol{D}_{1} \boldsymbol{D}_{1}^{T}\right)^{-1}
\end{aligned}
$$

where, $K_{N}$ is a positive null motion gain and $\boldsymbol{\delta}_{F}$ denotes the reference final gimbal angles. When (17) is applied to (7) and (13), the proposed method is expressed by

$$
\begin{aligned}
\dot{\boldsymbol{\eta}}_{\text {proposed }}= & \dot{\boldsymbol{\eta}}_{S D A}+\dot{\boldsymbol{\eta}}_{G F B} \\
= & {\left[\begin{array}{c}
\boldsymbol{W}_{s} \boldsymbol{D}_{0}^{T} \\
\boldsymbol{W}_{g} \boldsymbol{D}_{1 S D A}^{T}
\end{array}\right]\left[\boldsymbol{D}_{0} \boldsymbol{W}_{s} \boldsymbol{D}_{0}^{T}+\boldsymbol{D}_{1 S D A} \boldsymbol{W}_{g} \boldsymbol{D}_{1 S D A}{ }^{T}\right]^{-1} \boldsymbol{T}_{r} } \\
& +\left[\begin{array}{c}
\boldsymbol{0}_{4 \times 1} \\
K_{N} \boldsymbol{I}_{4 \times 4} \boldsymbol{S}\left(\boldsymbol{\delta}_{F}-\boldsymbol{\delta}\right)
\end{array}\right]
\end{aligned}
$$

The time $t=t_{2}$ is defined as the boundary point and null motion should activate after that. $K_{N}$ is set as

$$
K_{N}=0\left(t_{0} \leq t<t_{2}\right), K_{N} \neq 0\left(t_{2} \leq t\right) .
$$

The reference final gimbal angles $\boldsymbol{\delta}_{F}$ are selected on the condition that the Euclidean norm between the boundary point and each nomination expressed in (16) is a minimum. $\boldsymbol{\delta}_{F}$ is set at $t=t_{2}$ so as to satisfy the condition

$$
\begin{array}{lll}
\text { minimize } & f(m)=\left\|\boldsymbol{\delta}\left(t=t_{2}\right)-\boldsymbol{\delta}_{F}(m)\right\| \\
\text { subject to } & \boldsymbol{\delta}_{F}(m)=\left[\begin{array}{llll}
15 m & -15 m & 15 m & -15 m
\end{array}\right]^{T} .
\end{array}
$$

\section{Design of the rate profile of an attitude maneuver}

In terms of the gimbal angle feedback steering logic, the possibility of not converging to the reference angles by the intended time remains because null motion is not able to escape all types of singularity [10]. $\omega^{\text {profile }}$ is then designed to make the gimbal angular acceleration more gradual with the intention of having a longer period of gimbal angular feedback after the boundary point, as shown in Figure 7.

Supposing that $\omega_{\text {rmax }}$ is the maximum slew rate of the spacecraft and $\alpha$ is the angular acceleration, $\omega^{\text {profile }}$ can take either a triangular or trapezoidal shape depending on $\omega_{\text {rmax }}, \alpha$, and $\boldsymbol{\theta}^{\text {ref }}$. By taking a larger value of $\alpha$ in the period $t_{0} \leq t \leq t_{1}$, the duration of the attitude manoeuvre $t_{2} \leq t \leq t_{3}$ can be lengthened, according to

$$
\alpha^{\prime}=\gamma \alpha,
$$

where, the constant $\gamma \geq 1$.

\section{NUMERICAL SIMULATION}

\section{A. Simulation conditions}

The spacecraft manoeuvres around its roll axis from the initial attitude angles $\boldsymbol{\theta}\left(t_{0}\right)=\left[\begin{array}{lll}0 & 0 & 0\end{array}\right]^{T} \mathrm{deg}$ to the first reference attitude angles $\boldsymbol{\theta}^{\text {ref }}=\left[\begin{array}{lll}60 & 0 & 0\end{array}\right]^{T} \mathrm{deg}$, and then manoeuvres to the second attitude angles $\boldsymbol{\theta}^{\text {ref }}=\left[\begin{array}{lll}-15 & 0 & 0\end{array}\right]^{T} \mathrm{deg}$, which involves two rest-to-rest manoeuvres. Table 1 gives simulation parameters of the spacecraft and the VSCMGs. Table 2 gives design parameters of the proposed steering law.

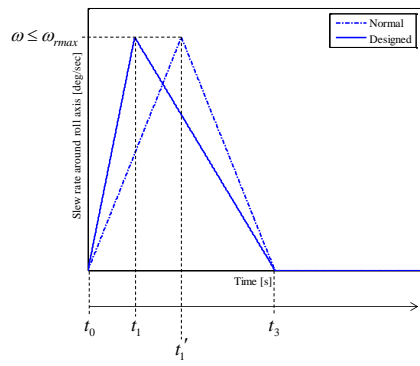

(a) Triangle type

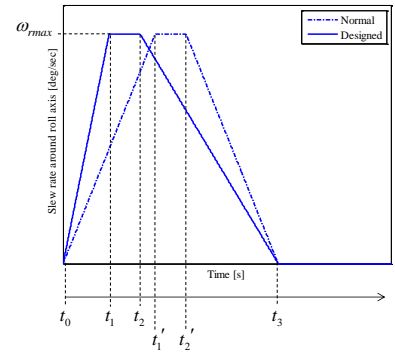

(b) Trapezoid type
Fig. 7. Designs of the slew rate profiles $\omega^{\text {profile }}$ of the spacecraft

The feedback controller of the attitude control system is a proportional derivative (PD) controller. In addition, the slew rate of the spacecraft and gimbal angular velocity of the VSCMGs when the attitude manoeuvre begins are supposed to be zero. To verify the validity of the rate profile design and gimbal angle feedback logic, the performances of three methods described in Table 3 are compared for the same feedback control system and the same mission. In Table 3, methods 1 and 2 are the comparative methods and method 3 is 
the proposed method. In method 1 , the reference final gimbal angles are set so that $\kappa_{s}$ has a minimum value. In method 2, $\omega^{\text {profile }}$ is not designed and a normal rest-to-rest manoeuvre is adopted.

\section{B. Simulation results}

Figures 8 and 9 show the results of the attitude manoeuvre and weighting value for each of the three methods, respectively.

TABLE. I. VSCMG AND SATELLITE PARAMETERS

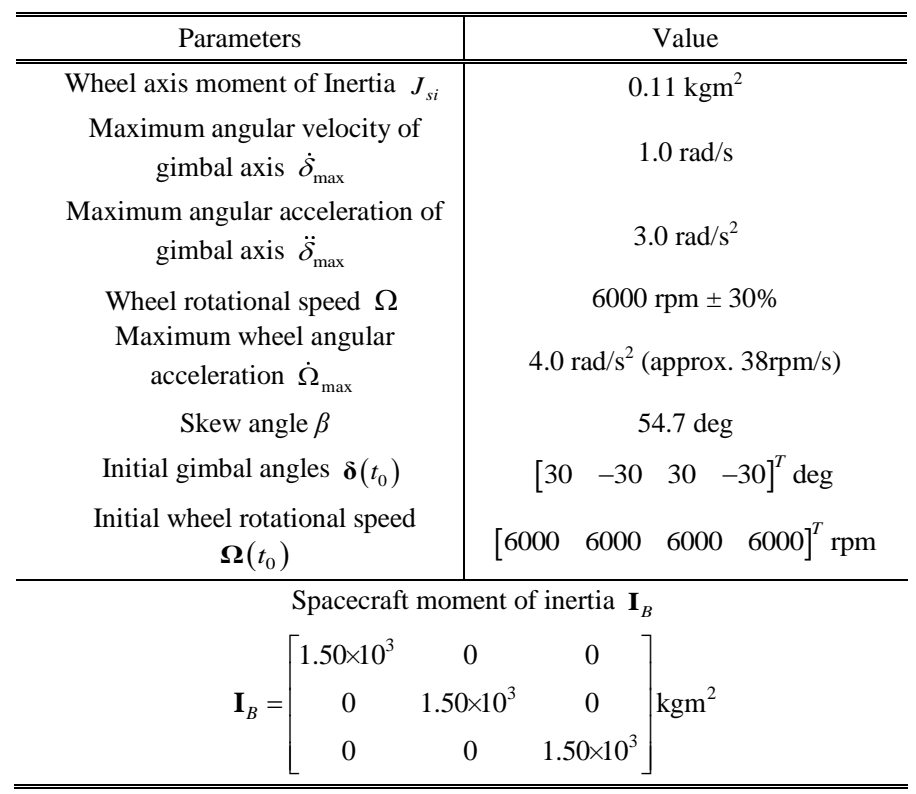

TABLE. II. DESIGN PARAMETERS

\begin{tabular}{l|l}
\hline \multicolumn{1}{c|}{ Steering law parameters } & \multicolumn{1}{c}{ Value } \\
\hline \hline$a$ & 1 \\
\hline$b$ & 1808 \\
\hline$c$ & 1.5 \\
\hline$\alpha_{0}$ & 0.05 \\
\hline$K_{N}$ & 0.5 \\
\hline \multicolumn{2}{|c}{$\mid$} \\
\hline Maneuver profile parameters & Value \\
\hline \hline$\omega_{r \text { max }}$ & $4.0 \mathrm{deg} / \mathrm{s}$ \\
\hline$\alpha$ & $0.36 \mathrm{deg} / \mathrm{s}^{2}$ \\
\hline$\gamma$ & 2.0 \\
\hline
\end{tabular}

In terms of the inner state of the VSCMGs, Figures 10, 11, and 12 show the results of the gimbal angles, singular index (condition number), and wheel rotational speed respectively. Figures 9 and 12 reveal that all methods can switch to the RW mode without oscillating the weighting values and without exceeding the range of the rated wheel rotational speed due to the design of weighting function in (9). In terms of the reference final gimbal angles, $\boldsymbol{\delta}_{F}=\left[\begin{array}{llll}30 & -30 & 30 & -30\end{array}\right]^{T}$ is set for both first and second manoeuvres in method 1 and $\boldsymbol{\delta}_{F}=\left[\begin{array}{llll}15 & -15 & 15 & -15\end{array}\right]^{T}$ is set for both first and second manoeuvres according to the calculation in (20) in methods 2 and 3 .

TABLE. III. SimULATION CONDITIONS FOR THE THREE METHODS

\begin{tabular}{|c||c|c|c|}
\hline & Method 1 & Method 2 & Method 3 \\
\hline \hline $\begin{array}{c}\text { CSCMG } \\
\text { steering law }\end{array}$ & \multicolumn{2}{|c|}{ SDA } \\
\hline $\begin{array}{c}\text { Gimbal angle } \\
\text { feedback }\end{array}$ & $t \geq t_{0}$ & \multicolumn{2}{|c|}{$t \geq t_{2}$} \\
\hline $\begin{array}{c}\text { reference } \\
\text { gimbal angles } \\
\boldsymbol{\delta}_{F}\end{array}$ & {$\left[\begin{array}{c}30 \\
-30 \\
30 \\
-30\end{array}\right]$} & {$\left[\begin{array}{c}15 m \\
-15 m \\
15 m \\
-15 m\end{array}\right], m=1$} \\
\hline $\begin{array}{c}\text { Maneuver } \\
\text { profile }\end{array}$ & Normal & Normal & Designed \\
\hline
\end{tabular}

Firstly, methods 1 and 3 are compared in terms of the difference in the reference gimbal angles. Figures 8(a) (c) and 9 (a)(c) reveal that methods 1 and 3 realise the reference attitude angles with approximately the same performance, switching the steering mode smoothly. By contrast, in the second manoeuvre of $t \geq 80$, Figures 10(a) and 11(a) reveal that gimbal angles in the final state of method 1 are in a singular state for $\boldsymbol{D}_{1}$ and gimbal angles of control moment gyros 1 and 2 are not able to converge although the gimbal angle feedback steering logic is active. Therefore, according to Figure 12(a), the wheel rotational speed of method 1 continuously changes even in the final state, which is not desirable for the saturation of the wheel rotational speed. By contrast in the case of method 3 from Figures 10(c) and 12(c), gimbal angles and wheel rotational speed can converge to the intended values of the final state in both first and second manoeuvres.

Secondly, methods 2 and 3 are compared in terms of gimbal behaviours. Figure 10(b) shows that method 2 fails to reach the reference final gimbal angles $\boldsymbol{\delta}_{F}=\left[\begin{array}{llll}15 & -15 & 15 & -15\end{array}\right]^{T}$ because the gimbal angle feedback steering logic does not work well. Whereas in the case of method 3, Figure 10(c) shows that the gimbal angle feedback steering logic works effectively and the proposed method reaches the reference angles owing to the design that the more gradual and longer latter manoeuvre. As a result, Figure 11(c) shows that method 3 succeeds in maintaining proper values of $\kappa_{s}$ and $\kappa_{g}$ of the final state in both first and second manoeuvres. The numerical simulation thus confirmed that the proposed method can realise the required attitude manoeuvre of the spacecraft while reaching the intended gimbal angles of the VSCMGs considering the distance from singularity in each final state of a single manoeuvre. 

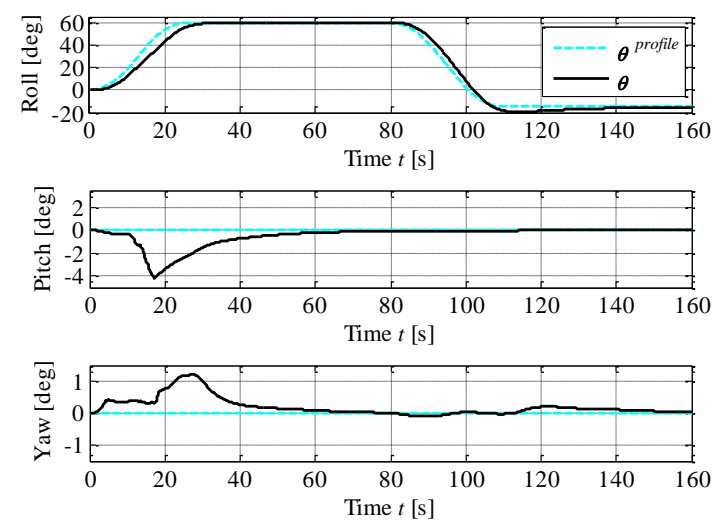

(a) Method 1
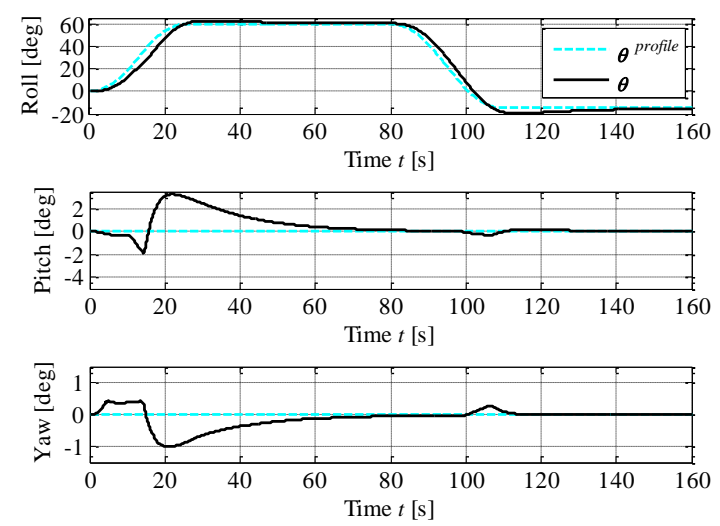

(b) Method 2
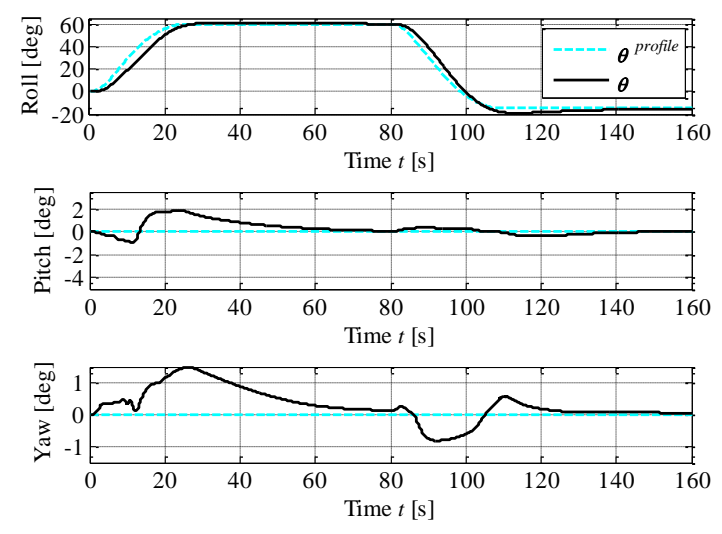

(c) Method 3

Fig. 8. Euler angle $\boldsymbol{\theta}$ of the spacecraft

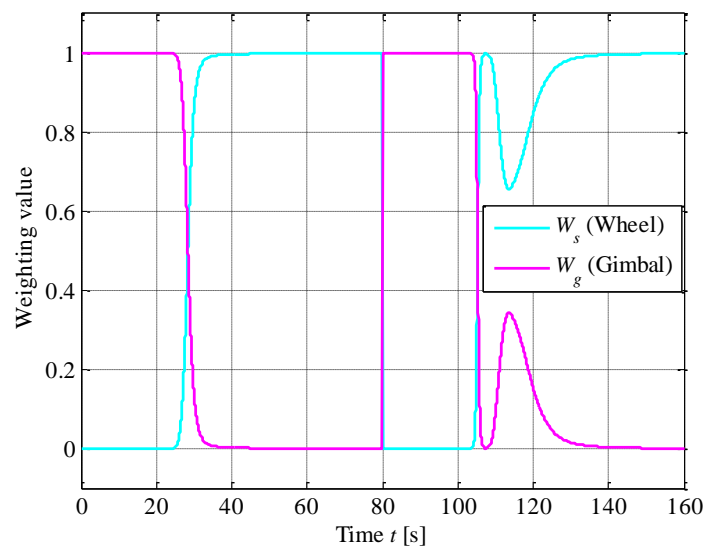

(a) Method 1

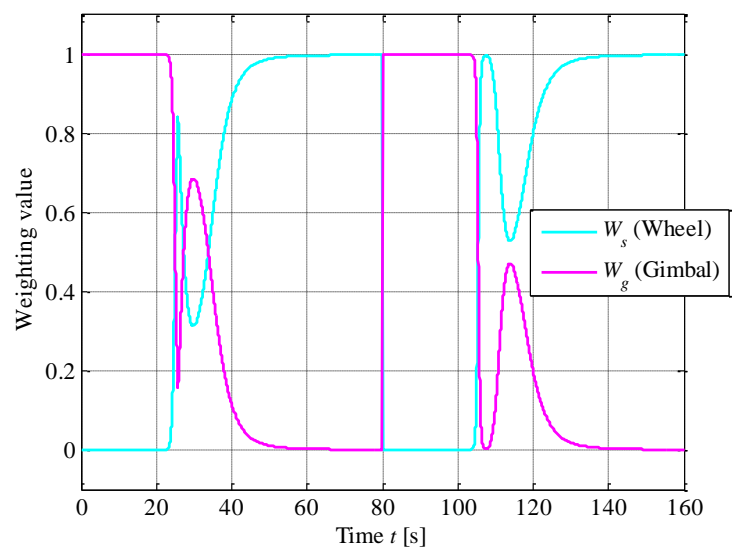

(b) Method 2

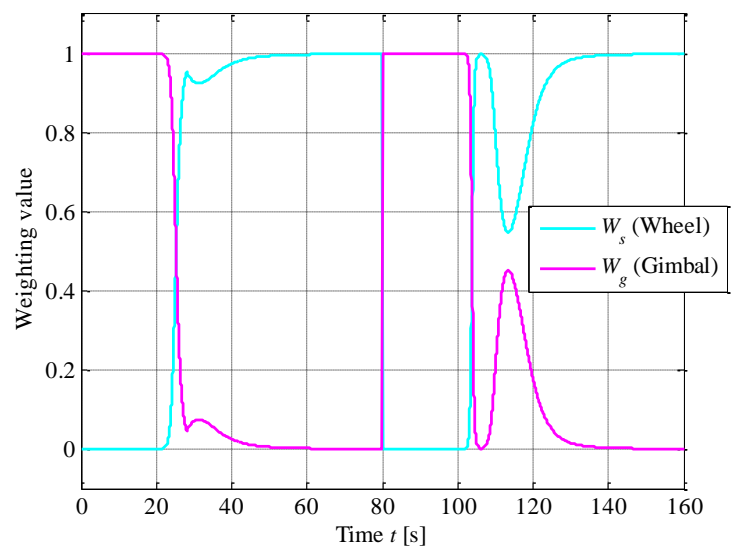

(c) Method 3

Fig. 9. Weighting value $W_{s}$ and $W_{g}$ 


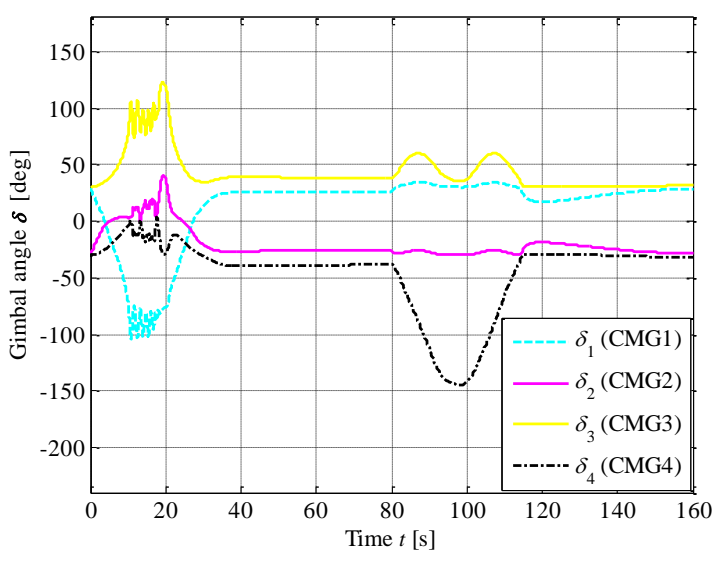

(a) Method 1

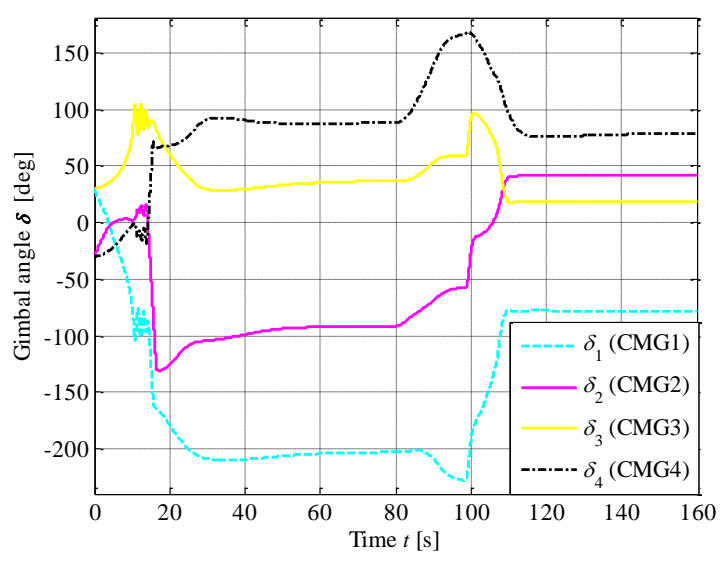

(b) Method 2

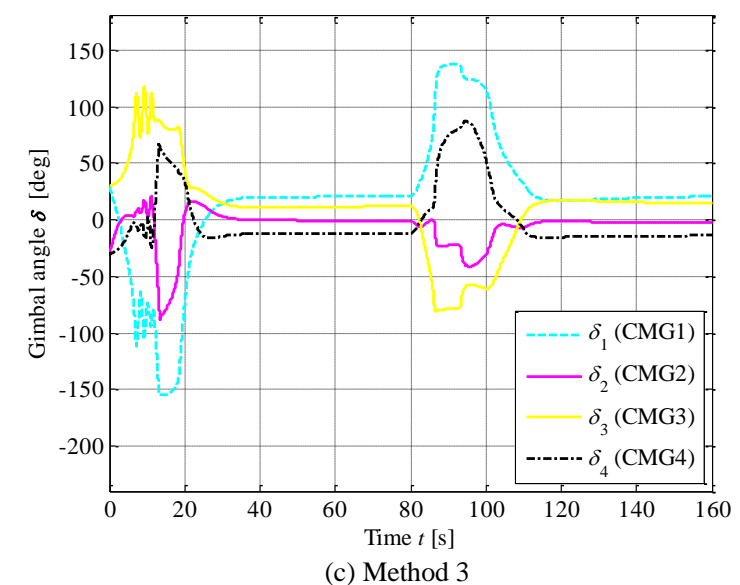

Fig. 10. Gimbal angles $\boldsymbol{\delta}$

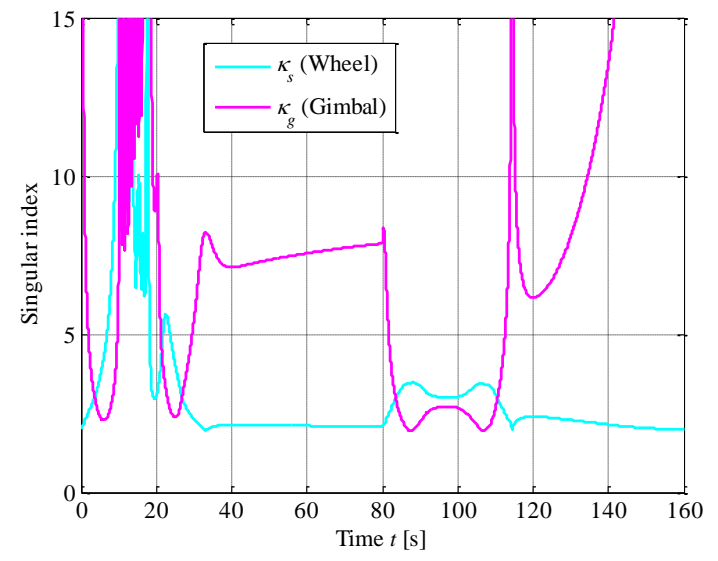

(a) Method 1

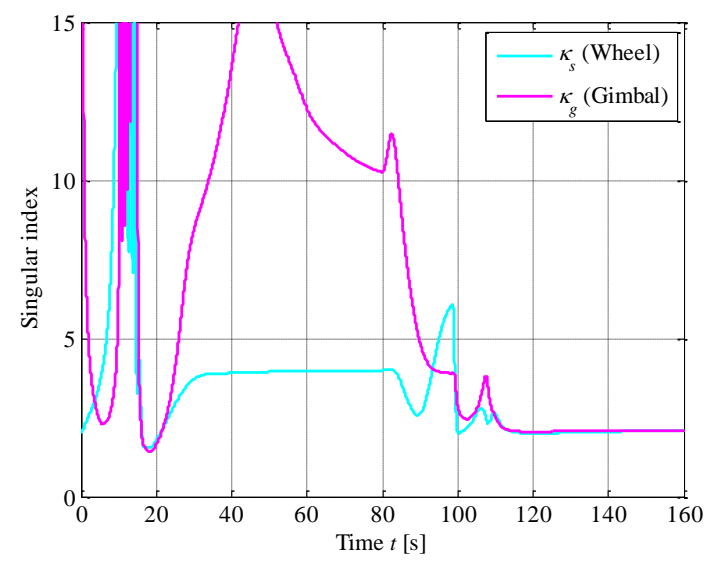

(b) Method 2

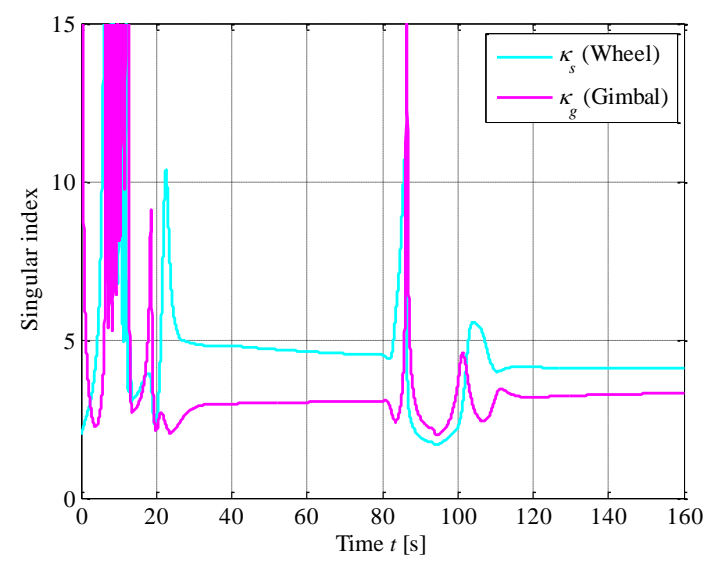

(c) Method 3

Fig. 11. Singular indices (condition numbers) $\kappa_{s}$ and $\kappa_{g}$ 


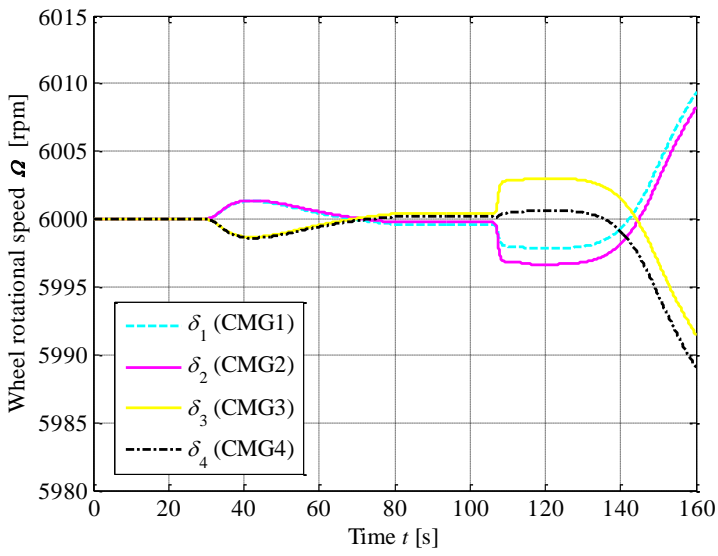

(a) Method 1

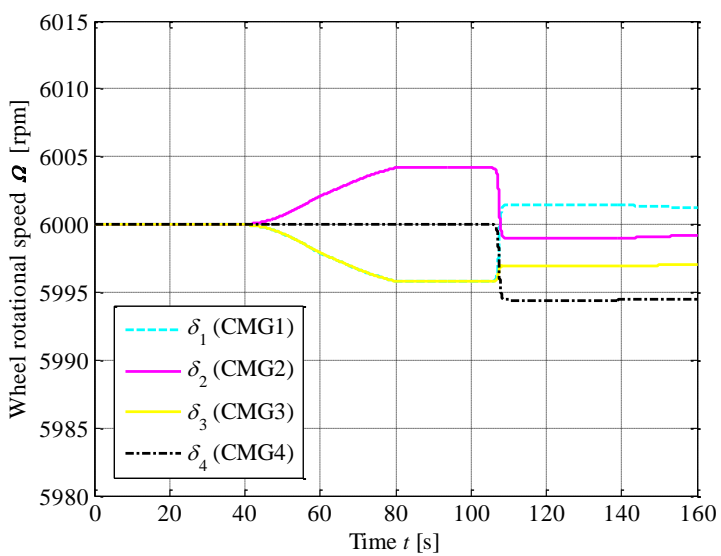

(b) Method 2

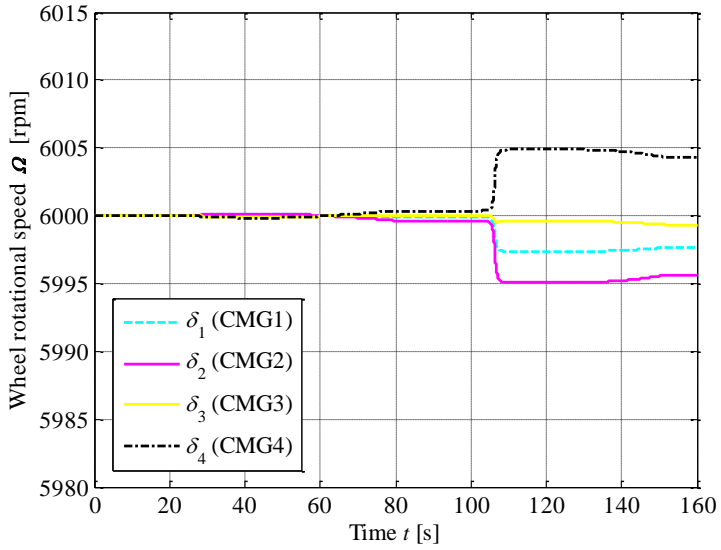

(c) Method 3

Fig. 12. Wheel rotational speed $\boldsymbol{\Omega}$

\section{CONCLUSION}

For the multi-target pointing and agile manoeuvring of a spacecraft with VSCMGs, the present study proposed a method of selecting reference final gimbal angles of a single manoeuvre and its application to the mode-scheduling steering law of VSCMGs. Firstly, the need to consider the condition numbers of both the CSCMG mode and RW mode in the final state of each single manoeuvre during consecutive manoeuvres was presented. In addition, the desired reference final gimbal angles of the single manoeuvre were introduced. Secondly, gimbal angle feedback steering logic using null motion was applied to the mode-scheduling steering law of VSCMGs to realise the reference final gimbal angles. To improve the reachability of the reference final gimbal angles, the boundary point is defined as the start time of deceleration in a rate profile of the spacecraft, and the nearest set of gimbal angles among nominated sets according to the Euclidean norm from the boundary point were selected as the reference final set at the middle of the manoeuvre. In addition, a rate profile was designed to make the gimbal angular acceleration more gradual with the intention of having a longer duration of gimbal angular feedback after the boundary point. The numerical simulation of consecutive rest-to-rest manoeuvres confirmed that the proposed method can realise the required attitude manoeuvre of the spacecraft while reaching the intended gimbal angles of the VSCMGs in each final state of a single manoeuvre.

As gimbal angle feedback steering logic using null motion in this study does not guarantee the complete reachability to the reference final gimbal angles, further verification and improvement through Monte Carlo simulation etc. assuming various manoeuvre cases are future work.

\section{REFERENCES}

[1] Leve, F. A., Brian, J. H., and Mason, A. P., "Spacecraft Momentum Control Systems", Springer Space Technology Library, vol. 1010, pp.151-152, 2015.

[2] D. Verbin, V.J. Lappas, "Rapid rotational maneuvering of rigid satellites with hybrid actuators configuration," J. Guid. Control. Dyn., vol. 36, pp. 532-547, 2013.

[3] B. Wie, D. Bailey, C. Heiberg, "Rapid Multitarget Acquisition and Pointing Control of Agile Spacecraft," J. Guid. Control. Dyn., vol. 25, pp. 96-104, 2002.

[4] V. Lappas, D. Richie, C. Hall, J. Fausz, B. Wilson, "Survey of Technology Developments in Flywheel Attitude Control and Energy Storage Systems," J. Guid. Control. Dyn., vol. 32, pp. 354-365, 2009.

[5] D.J. Richie, V.J. Lappas, B. Wie, "Practical Steering Law for Small Satellite Energy Storage and Attitude Control,” J. Guid. Control. Dyn., vol. 32, pp. 1898-1911, 2009.

[6] M. Takahashi, Y. Nanamori, K. Yoshida, "Rapid Multi-target Pointing and High Accuracy Attitude Control Steering Law of Variable Speed Control Moment Gyroscopes," AIAA Guid. Navig. Control Conf. Exhib., AIAA 2008-7015, 2008.

[7] S. Kasai, H. Kojima, "Gain-scheduled Steering Control Law for Variable Speed Control Moment Gyros," AIAA Guid. Navig. Control Conf. Exhib., AIAA 2013-4796, 2013.

[8] S.R. Vadali, S. Krishna, "Suboptimal Command Generation for Control Moment Gyroscopes and Feedback Control of Spacecraft," J. Guid. Control. Dyn., vol. 18, pp. 1350-1354, 1995.

[9] H. Kurokawa, "Survey of Theory and Steering Laws of Single-Gimbal Control Moment Gyros," J. Guid. Control. Dyn., vol. 30, pp. 1331-1340, 2007.

[10] F. A. Leve, N.G. Fitz-Coy, "Hybrid Steering Logic for Single-Gimbal Control Moment Gyroscopes," J. Guid. Control. Dyn., vol. 33, pp. 12021212, 2010.

[11] K.A. Ford, C.D. Hall, "Flexible spacecraft reorientations using gimbaled momentum wheels," Adv. Astronaut. Sci. 97 PART 2, pp. 1895-1913, 1997.

[12] Zhao, Hui, Feng Liu, and Yu Yao, "Optimization design steering law for VSCMGs with the function of attitude control and energy storage", Aerosp. Sci. Technol., vol. 65, pp. 9-17, 2017.

[13] H. Yoon, P. Tsiotras, "Singularity Analysis of Variable Speed Control Moment Gyros,” J. Guid. Control. Dyn., vol. 27, pp. 374-386, 2004. 
[14] H. Schaub, S.R. Vadali, J.L. Junkins, H. Schaub, S.R. Vadali, J.L. Junkins, "Feedback Control Law for Variable Speed Control Moment Gyros," J. Astronaut. Sci., vol. 46, pp. 307-328, 1998.

[15] D.R. Nazareth S. Bedrossian, Joseph Paradiso, Edward V. Bergmann, "Redundant single gimbal control moment gyroscope singularity analysis," J. Guid. Control. Dyn., vol. 13, pp. 1096-1101, 1990.
[16] Liang, T., and Shijie, X., "Integrated power and attitude control using VSCMGs for agile satellite," Automatic Control in Aerospace, pp. 167172, 2004.

[17] K.A. Ford, C.D. Hall, "Singular direction avoidance steering for controlmoment gyros," J. Guid. Control. Dyn., vol. 23, pp. 648-656, 2000. 